Clinical experience suggests that the major risk of bleeding is determined by the presence of damaged vessels, with the necessity to form haemostatic plugs. Search for any possible vascular damage is, therefore, more important than any change in the haematological findings when predicting the risk. Surgical wounds older than 72 hours and healing by first intention do not appear to create a hazard but bleeding may come from granulating areas or from around drainage tubes in wounds much older than this.

\section{Conclusions}

The real need for treatment with streptokinase is confined to those patients with thrombi in the popliteal or more proximal deep veins, where there is a great risk of pulmonary embolism. Early treatment is more likely to preserve the function of venous valves. Treatment may be contraindicated by vessel damage which requires the continued formation of haemostatic plugs. The selection of these patients is, unfortunately, impossible on clinical grounds alone. It requires phlebography or scanning to detect the uptake of radioactive labelled-fibrinogen.

This communication has so far ignored the other important question, the occasional failures of treatment. Clinical impressions suggest that these are often related to the age of the thrombus or to the continuation of a recognizable stimulus to thrombosis. There is no absolute rule but thrombi present for longer than five days often fail to dissolve. Rethrombosis has been an important problem in patients with pelvic sepsis. The majority of reported studies have used a high loading dose of streptokinase with a profound fall in plasminogen; further work is needed to compare these results with those of other dose regimes.

\section{Abstract}

Thrombolytic Treatment of Recent Arterial Occlusion M. VERSTRAETE (Academisch Ziekenhuis St Raffel, Leuven, Belgium)

It has been shown conclusively that during the administration of streptokinase (SK) in patients with a recent thromboembolic occlusion of a limb artery, patency of the main artery can be restored (Verstraete, Amery, and Vermylen, 1963; Winckelmann, Hiemeyer, Weissleder, and Schoop, 1963).

The results of 15 European centres which are using purified streptokinase administered intravenously for the thrombolytic treatment of recent and acute occlusions in limb arteries have been collected by Hess (1967). The dose of streptokinase infused was usually the titrated initial dose followed by a maintenance dose of 100000 units streptokinase per hour. This study was not planned ahead but the results of the various centres were collected, assuming that the patient's condition, the dosage scheme, and the criteria of evaluation were similar.

In total 252 arterial emboli and 206 thrombi or unclassified arterial occlusions were treated. A return of arterial pulsations at all levels was obtained in $43 \%$, partial clearing (ie, return of arterial pulsations at least at one level but not at all levels) was obtained in $16 \%$, and there were $41 \%$ failures.

A higher success rate was obtained for arterial occlusions at the popliteal level $(61 \%)$ compared to aortic occlusion (12\%); this difference is statistically significant $(P=0.002)$. The thrombolytic success rate was greater in patients with arterial emboli $(55 \%)$ compared with arterial thrombosis (34\%). Also this difference is statistically significant $(P<0.001)$ and persists in comparing groups of arterial thrombi or emboli of different duration (between 24 and 120 hours). The sooner an arterial occlusion is treated with streptokinase, the shorter the infusion period required to obtain thrombolysis: emboli of less than 19 hours' duration had an average lysis time of 39 hours, emboli older than 19 hours' duration required an average of 62 hours' treatment; a parallel relationship was found for arterial thrombosis. When therapy was started within 30 hours, lysis occurred on the average in seven hours; when therapy was started after 30 hours, lysis occurred in about 99 hours (Schmutzler, 1968).

Two important side effects should be mentioned: the first is the risk of emboli during thrombolytic therapy: $3 \cdot 1 \%$ of all patients developed a non-fatal embolus and $2 \cdot 3 \%$ developed a fatal embolus. 
Secondly, the incidence of bleeding was $4.9 \%$ amongst which seven fatal cerebral haemorrhages occurred.

However, neither the incidence of spontaneous disobliteration nor the optimal dose and duration of SK therapy have yet been established. Furthermore, no controlled and randomized study is available comparing in this condition the value of SK treatment to other current therapeutic regimens.

Since the introduction of Fogarty's catheter (Fogarty, Cranley, Krause, Strasser, and Hafner, 1963) the possibilities of vascular surgery have been extended, and this method represents an important alternative for the treatment of patients with an arterial embolism and to minor extent arterial thrombosis in atherosclerotic arteries. There is therefore an urgent need to organize a well designed cooperative trial comparing the immediate and late outcome of recent occlusions of limb arteries treated with heparin, Fogarty catheter, streptokinase, or urokinase in homogenous groups of patients allocated at random to one of four treatment schedules. Separate groups should be made for arterial emboli and arterial thrombosis in young patients.

\title{
Treatment of chronic arterial occlusions with streptokinase
}

\author{
H. POLIWODA
}

From Abtlg. Hamatologie, Dept. Innere Med., Hoschschule, Hanover, Germany

Previous studies have demonstrated that fibrinolytic therapy with streptokinase is successful in patients with acute arterial occlusion. However, this form of therapy is generally considered to be ineffective in the treatment of longstanding arterial occlusions, in which conservative therapeutic efforts have been directed mainly at the establishment of collateral circulation and not at establishing flow in the occluded vessel itself.

Gottlob, Blümel, Piza Brücke, and Böhmig (1968), in experiments in vitro, demonstrated lysis of thrombi removed from patients with chronic arterial occlusions. Encouraged by these results, Schoop, Martin, and Zeitler (1968), Ehringer and Fischer (1968), and our own group started clinical investigations into the thrombolytic treatment of chronic arterial occlusions (Poliwoda, Alexander, Buhl, Holsten, and Wagner, 1969).

\section{Patients}

One hundred and thirty-two patients (118 men and 14 women, 36 to 77 years of age) were treated with streptokinase. All had longstanding arterial occlu- sion, the duration of the disease as indicated by intermittent claudication varying from four weeks to 16 years. In all patients, the clinical findings were supported by oscillography, light plethysmography, and arteriography. These investigations were performed before and after streptokinase therapy, and the results compared.

\section{Therapeutic Programme}

The initial dose of streptokinase was determined by the streptokinase resistance test. The enzyme was given by intravenous infusion over a period of 20 minutes and followed by a dose of 125000 units per hour for 16 hours by continuous intravenous infusion. Therapy was interrupted for eight hours during the night. The effect of streptokinase therapy was controlled by the thrombin time (normal 18 to 20 seconds). A twofold to threefold prolongation of the normal value was considered optimal. After the third day the thrombin time usually returned towards normal (less than 35 seconds) and treatment with heparin was begun, administered by continuous intravenous infusion, in a dose of 30000 units over 